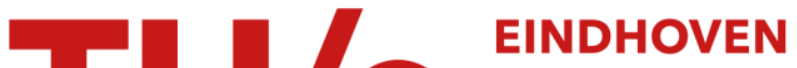 \\ UNIVERSITY OF \\ TECHNOLOGY
}

\section{Scale space representations locally adapted to the geometry of base and target manifold}

Citation for published version (APA):

Florack, L. M. J. (2010). Scale space representations locally adapted to the geometry of base and target manifold. (CASA-report; Vol. 1063). Technische Universiteit Eindhoven.

Document status and date:

Published: 01/01/2010

\section{Document Version:}

Publisher's PDF, also known as Version of Record (includes final page, issue and volume numbers)

\section{Please check the document version of this publication:}

- A submitted manuscript is the version of the article upon submission and before peer-review. There can be important differences between the submitted version and the official published version of record. People interested in the research are advised to contact the author for the final version of the publication, or visit the $\mathrm{DOI}$ to the publisher's website.

- The final author version and the galley proof are versions of the publication after peer review.

- The final published version features the final layout of the paper including the volume, issue and page numbers.

Link to publication

\section{General rights}

Copyright and moral rights for the publications made accessible in the public portal are retained by the authors and/or other copyright owners and it is a condition of accessing publications that users recognise and abide by the legal requirements associated with these rights.

- Users may download and print one copy of any publication from the public portal for the purpose of private study or research.

- You may not further distribute the material or use it for any profit-making activity or commercial gain

- You may freely distribute the URL identifying the publication in the public portal.

If the publication is distributed under the terms of Article 25fa of the Dutch Copyright Act, indicated by the "Taverne" license above, please follow below link for the End User Agreement:

www.tue.nl/taverne

Take down policy

If you believe that this document breaches copyright please contact us at:

openaccess@tue.nl

providing details and we will investigate your claim. 


\section{EINDHOVEN UNIVERSITY OF TECHNOLOGY}

Department of Mathematics and Computer Science

CASA-Report Io-63

October 2010

Scale space representations locally adapted to the geometry of base and target manifold

by

L.M.J. Florack

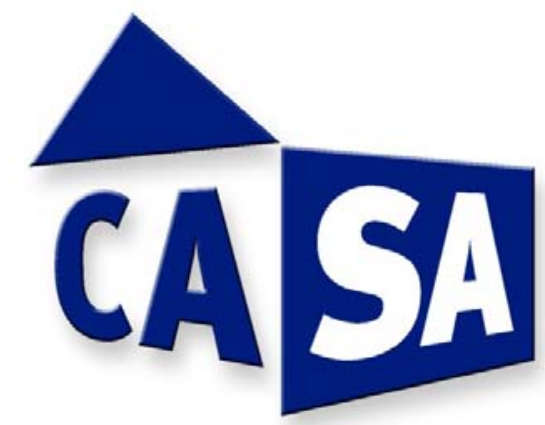

Centre for Analysis, Scientific computing and Applications

Department of Mathematics and Computer Science

Eindhoven University of Technology

P.O. Box 513

5600 MB Eindhoven, The Netherlands

ISSN: 0926-4507 



\title{
Scale Space Representations Locally Adapted to the Geometry of Base and Target Manifold
}

\author{
Luc Florack
}

October 25, 2010

\begin{abstract}
We generalize the Gaussian multi-resolution image paradigm for a Euclidean domain to general Riemannian base manifolds and also account for the codomain by considering the extension into a fibre bundle structure. We elaborate on aspects of parametrization and gauge, as these are important in practical applications. We subsequently scrutinize two examples that are of interest in bio-mathematical modeling, viz. scale space on the unit sphere, used among others for codomain regularization in the context of high angular resolution diffusion imaging (HARDI), and retino-cortical scale space, proposed as a biologically plausible model of the human visual pathway from retina to striate cortex.
\end{abstract}

\section{Introduction}

Physical field observables (referred to as signals or images henceforth) have an intrinsically limited resolution. Resolution invariance can be realized (for levels below measurement resolution) by generating a one-parameter family of gracefully degraded copies of the fiducial image in a causal fashion, subject to certain invariance axioms, of which scale invariance is the one most relevant in this context. The canonical way to operationalize this is by convolution with a normalized Gaussian, yielding a so-called Gaussian scale space [8, 13, 16, 21, 27]. Traditionally this is done against a Euclidean background geometry, and in a homogeneous and isotropic fashion. This also holds for certain generalizations proposed in the literature, such as the so-called $\alpha$-scale spaces by Duits et al. [7], the relativistic scale spaces by Burgeth et al. [4], etc. Moreover, scale space images are viewed as smooth functions with a globally defined codomain. That is to say, image values are expressed relative to a universal standard (unit of intensity), which implies that they can be compared irrespective of the base point to which they are attached.

Local adaptation mechanisms have been proposed to control the resolution degradation process locally so as to account for the differential structure of the image, e.g. for the purpose of "edge enhancement" [12, 24, 31]. Such mechanisms rely on and make use of the specific local structure of the image function, and always yield nonlinear filtering paradigms.

Here we consider local adaptation mechanisms of a different kind, viz. based on the geometry of the domain and codomain of the image. We depart from the (typically implicit) assumption that space is Euclidean, and even from the assumption that the image can be modeled as a function from a spatial, temporal, or spatiotemporal domain into a globally defined codomain. Instead we adapt the canonical paradigm to a general (Riemannian) geometry of its domain, and regard the codomain as a point-wise entity, leading to a so-called fibre bundle. Linearity is manifest throughout.

\section{Theory}

\subsection{Preliminaries on Riemannian Geometry}

Let $S: \Omega^{m} \rightarrow \mathbb{R}$ denote a given raw signal defined on an $m$-dimensional Riemannian surface $\Omega^{m}$, say. This could be a surface embedded in an $n$-dimensional Euclidean space, in which case it inherits its (generally non-flat) 
Riemannian metric from that of the embedding space. In this case we may parametrize $\Omega^{m}$ using coordinates $\xi^{\mu}$, $\mu=1, \ldots, m \leq n$, and derive the components of the metric tensor of $\Omega^{m}$ from that of $\mathbb{R}^{n}$ as follows:

$$
g_{\mu v}=\frac{\partial x^{i}}{\partial \xi^{\mu}} \eta_{i j} \frac{\partial x^{j}}{\partial \xi^{v}},
$$

in which $\eta_{i j}, 1 \leq i, j \leq n$, denote the components of the Euclidean metric tensor of the embedding space, and $g_{\mu \nu}, 1 \leq \mu, v \leq m$ the ones induced on $\Omega^{m}$. For notational convenience we set $g=\operatorname{det} g_{\mu \nu}$ and $\eta=\operatorname{det} \eta_{i j}$. These are relative scalars. More precisely, $\sqrt{g} d \xi^{1} \ldots d \xi^{m}$ and $\sqrt{\eta} d x^{1} \ldots d x^{n}$ are the basic volume elements on $\Omega^{m}$, respectively $\mathbb{R}^{n}$. Henceforth we will concentrate on the intrinsic properties of $\Omega^{m}$ without reference to its embedding in $\mathbb{R}^{n}$. In fact, no such embedding will be required.

By $D_{\mu}$ we shall denote the covariant derivative with respect to $x^{\mu}$ induced by the metric tensor $g_{\mu \nu}$. By construction the metric is "covariantly constant": $D_{\rho} g_{\mu v}=0$, whence also $D_{\mu} g=0$. (Under the additional assumption that space is free of so-called torsion, one can take this as the definition of the covariant derivative [26].) The covariant derivative defines a connection that allows us to compare neighbouring quantities despite locally varying (an)holonomic bases by "correcting" for the rate of change of the basis vectors in any direction along the base manifold. If $\left\{\mathbf{e}_{\mu}\right\}$ is a basis of the local tangent space at some implicit point of $\Omega^{m}$, then by definition

$$
D_{v} \mathbf{e}_{\mu}=\Gamma_{\mu \nu}^{\rho} \mathbf{e}_{\rho} .
$$

The coefficients can be constructed so as to be "compatible with the metric", i.e. so as to respect the requirement of covariant constancy. "Metric compatibility" is tantamount to the applicability of the product rule despite a spatially varying metric. That is, if $\mathbf{v}, \mathbf{w}$ are differentiable vector fields with components $v^{\mu}, w^{\mu}$ relative to the local basis $\left\{\mathbf{e}_{\mu}\right\}$, then

$$
\nabla(\mathbf{v}, \mathbf{w})=(\nabla \mathbf{v}, \mathbf{w})+(\mathbf{v}, \nabla \mathbf{w}),
$$

or, in terms of components,

$$
D_{\rho}\left(g_{\mu \nu} v^{\mu} w^{v}\right)=D_{\rho} v^{\mu} g_{\mu \nu} w^{v}+v^{\mu} g_{\mu v} D_{\rho} w^{v} .
$$

This uniquely establishes the (symmetric part of the) so-called Christoffel symbols in Eq. (2):

$$
\Gamma_{\mu v}^{\rho}=\frac{1}{2} g^{\rho \lambda}\left(\partial_{\mu} g_{\lambda v}+\partial_{v} g_{\lambda \mu}-\partial_{\lambda} g_{\mu v}\right) .
$$

The components of $\nabla \mathbf{v}$ are given by

$$
D_{\mu} v^{v}=\partial_{\mu} v^{v}+\Gamma_{\rho \mu}^{v} v^{\rho} .
$$

(It can be shown that the antisymmetric part of the Christoffel symbols captures the torsion of the underlying manifold [26], which, in our case, will be assumed to vanish.)

Formulae for the component representation of $\nabla \mathbf{T}$ for any type of tensor field $\mathbf{T}$ are now uniquely determined by application of the product rule using covariant constancy of the metric in a similar fashion, together with the observation that $\nabla f$ coincides with the familiar gradient if $f$ is a scalar function (no " $\Gamma$-corrections" need to be carried out since function values are absolute, i.e. do not rely on a local basis). Just fill in the (co-)vector slots of $\mathbf{T}$ so as to produce a scalar field, and differentiate. In particular, if $\omega$ is a covector with components $\omega_{\mu}$ relative to a local basis $\left\{\mathbf{e}^{\mu}\right\}$ of the dual tangent space, then the components of $\nabla \omega$ are obtained by working out the identity $\nabla(\omega(\mathbf{v}))=\nabla \omega(\mathbf{v})+\omega(\nabla \mathbf{v})$ in terms of components. As a result one finds

$$
D_{\mu} \omega_{v}=\partial_{\mu} \omega_{v}-\Gamma_{v \mu}^{\rho} \omega_{\rho} .
$$

For further details on Riemannian geometry and tensor calculus the reader is referred to the literature [26].

\subsection{Scale Space}

The above geometric elaboration allows us to extend the scale space paradigm beyond the usual, Euclidean context $[8,13,16,21,27]$. To this end, consider the following functional, in which (the minimizer) $S_{t}: \Omega^{m} \rightarrow \mathbb{R}$ will be seen to act as the scale space extension of the raw signal $S$ :

$$
E\left(S_{t}\right)=\int_{\Omega^{m}}\left[\left(S(\xi)-S_{t}(\xi)\right)^{2}+\sum_{k \geq 1} \frac{t^{k}}{k !} D_{\mu_{1}} \ldots D_{\mu_{k}} S_{t}(\xi) D^{\mu_{1}} \ldots D^{\mu_{k}} S_{t}(\xi)\right] D \xi .
$$


Here, $D^{\mu} S_{t}=g^{\mu v} D_{v} S_{t}$, and $D \xi=\sqrt{g} d \xi^{1} \ldots d \xi^{m}$ is a parametrization invariant measure. Equivalently, one may write (notice the appearance of minus signs)

$$
E\left(S_{t}\right)=\int_{\Omega^{m}}\left[\left(S(\xi)-S_{t}(\xi)\right)^{2}+\sum_{k \geq 1} \frac{(-t)^{k}}{k !} S_{t}(\xi) \Delta_{g}^{k} S_{t}(\xi)\right] D \xi
$$

in which $\Delta_{g}=g^{\mu v} D_{\mu} D_{v}$ denotes the self-adjoint Laplace-Beltrami operator on $\Omega^{m}$, i.e. if $f: \Omega^{m} \rightarrow \mathbb{R}$ is a scalar function on $\Omega^{m}$, then we have

$$
\Delta_{g} f=\frac{1}{\sqrt{g}} \partial_{\mu}\left(g^{\mu v} \sqrt{g} \partial_{v} f\right)
$$

Using covariant constancy of the metric, and the fact that $\Delta_{g}^{k}$ is self-adjoint for any $k=\mathbb{Z}_{0}^{+}$, it is straightforward to derive the corresponding Euler-Lagrange equations. For Eq. (8) this yields an "infinite order" PDE for the family $S_{t}, t \geq 0$, given the data function $S$ :

$$
S=\sum_{k \geq 0} \frac{(-t)^{k}}{k !} \Delta_{g}^{k} S_{t}=\exp \left(-t \Delta_{g}\right) S_{t}
$$

Inversion yields the solution ${ }^{1}$

$$
S_{t}=\sum_{k \geq 0} \frac{t^{k}}{k !} \Delta_{g}^{k} S=\exp \left(t \Delta_{g}\right) S .
$$

Notice that it satisfies the heat equation on $\Omega^{m}$ :

$$
\partial_{t} S_{t}-\Delta_{g} S_{t}=0
$$

with initial condition $S_{0}=S$. This is analogous to the standard scale space representation in the Euclidean plane, with the Euclidean Laplace operator $\Delta$ on $\mathbb{R}^{n}$ formally replaced by $\Delta_{g}$. The parameter $t \geq 0$ controls the (inverse) resolution.

The asymptotic cases are as follows:

$$
\begin{aligned}
\lim _{t \rightarrow 0^{+}} S_{t} & =S, \\
\lim _{t \rightarrow \infty} S_{t} & =\frac{\int_{\Omega^{m}} S(\xi) d \xi}{\int_{\Omega^{m}} d \xi} .
\end{aligned}
$$

The latter expression holds for compact $\Omega^{m}$, but can be generalized with some care. The physical significance of $S_{t}$ is that it represents the raw data $S$ resolved at finite scale $t \in(0, \infty)$. For this reason $S_{t}$ is referred to as the scale space representation of $S$.

\subsection{Parametrization Aspects}

In practice one must account for parametrization aspects. For instance, the assumption of linearity typically fails to hold due to nonlinear signal transfer in real physical or biological systems. Typically such systems are characterized by a compact or semi-infinite range, which is obviously inconsistent with linearity. Nevertheless, linearity implies no loss of generality in cases that can be understood in terms of codomain mappings of the type $S_{t}=\gamma\left(U_{t}\right)$ for some monotonic function $\gamma: \mathbb{R} \rightarrow \mathbb{R}$, with $\gamma^{\prime}>0$, say. Recall that the incorporation of a general Riemannian metric instead of a Euclidean one may likewise account for a physically or biologically relevant reparametrization of the base manifold, or, more generally, of a Riemannian metric transform (which may or may not be induced by a domain reparametrization). As a result one should study a modification of the basic paradigm, Eq. (13), by instead considering

$$
\partial_{t} U_{t}-\Delta_{g} U_{t}-\mu\left\|\nabla U_{t}\right\|_{g}^{2}=0
$$

in which $\mu=\left(\ln \gamma^{\prime}\right)^{\prime}$ and $\|\nabla u\|_{g}^{2}=g^{\mu v} \partial_{\mu} u \partial_{\nu} u$.

\footnotetext{
${ }^{1}$ The operator $\exp \left(t \Delta_{g}\right)$ is bounded and defines a strongly continuous semigroup for $t \in \mathbb{R}^{+} \cup\{0\}$.
} 


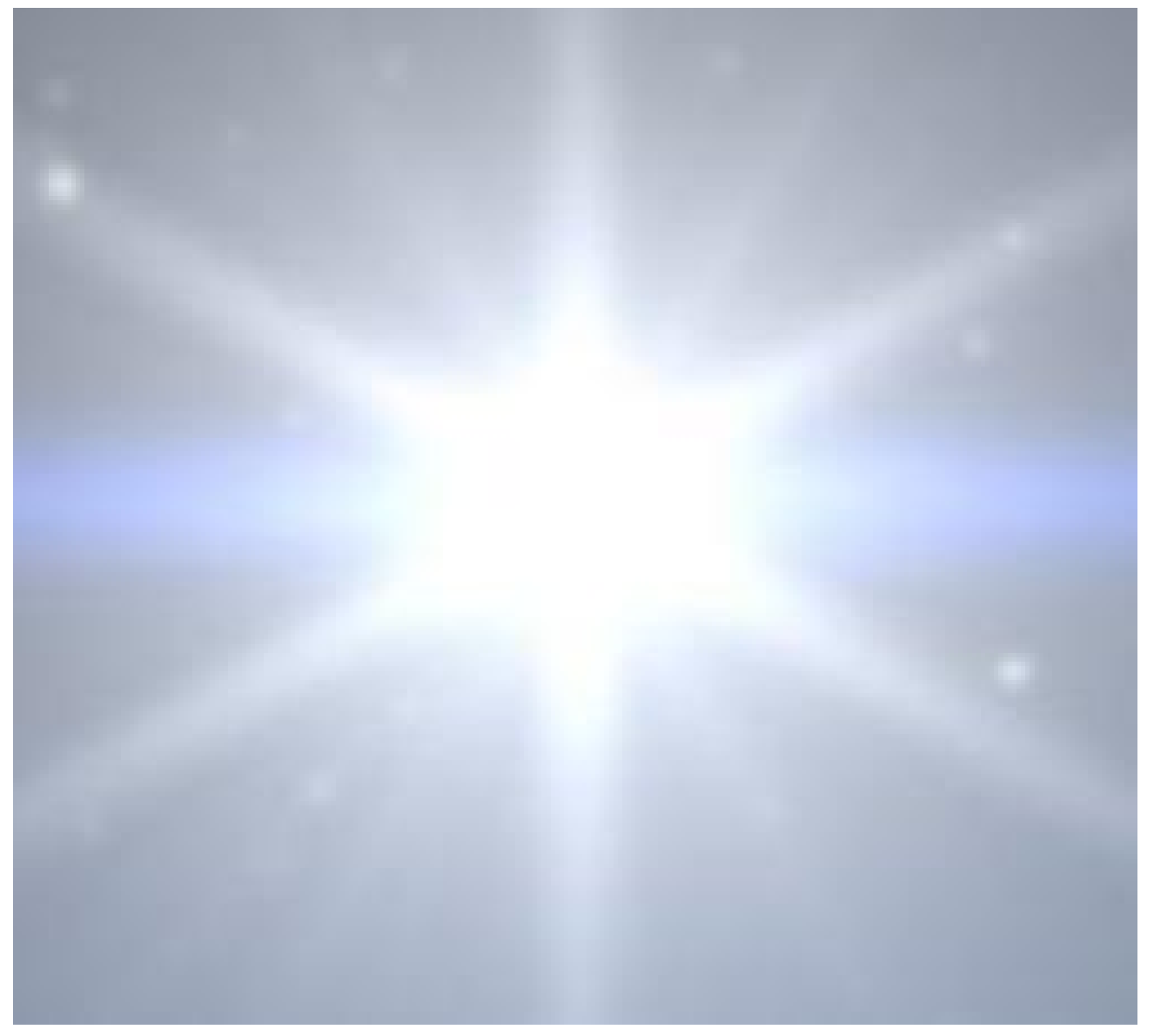

Figure 1: Bright sun illusion. The sun appears brighter than the paper background. Are we deceived because of our bias to recognize the bright object in the middle as the sun, or as a light bulb?

\subsection{Gauge Aspects}

A more subtle aspect is that of gauging signal values obtained at different locations on the base manifold. This aspect has received virtually no attention in the image processing literature, with a few notable exceptions [11, $18,19]$. Especially in biological vision it is not self-evident that signals of equal physical magnitude at different locations in the visual field are perceived as such, i.e. have the same perceptual magnitude. Indeed, there are well-known illusions refuting the validity of this ubiquitous assumption, such as the "bright sun" illusion, Fig. 1, and the Craik-O'Brien-Cornsweet illusion, Fig. 2.

Illusions such as those of Figs. 1-2 cannot be understood if we insist on treating the signal as a spatial function. Instead, we must somehow gauge its values by selecting appropriate perceptual units of intensity pointwise. Georgiev argued that this can be achieved by modeling the signal as a section of a fibred space [11]. Koenderink exploited the same idea in an image processing context [19]. Each fibre represents the local codomain of possible intensity values at one point of the image domain. The "vertical" projection of a fibre onto the base manifold is well-defined, and yields the base point to which it is attached. In this view, however, one declines from a "horizontal" projection of intensity values, i.e. from the possibility to compare signal values at distinct locations. Instead, one furnishes the fibred space with a so-called connection, to be defined below, which in turn induces a gauge field that allows us to "correct" for differences of neighbouring signal values by accounting for the spatial variation of the perceptual units of intensity.

The heuristic argument above suggests that instead of the raw, physical value $S(x)$ of the signal function we consider its value relative to the local unit of intensity, $\sigma(x)$ say, i.e. we consider perceived intensity $S(x) \sigma(x)$ for some perceptual (locally adaptive) unit $\sigma(x)$. This selection of a local unit, $\sigma(x)$, respectively any local 

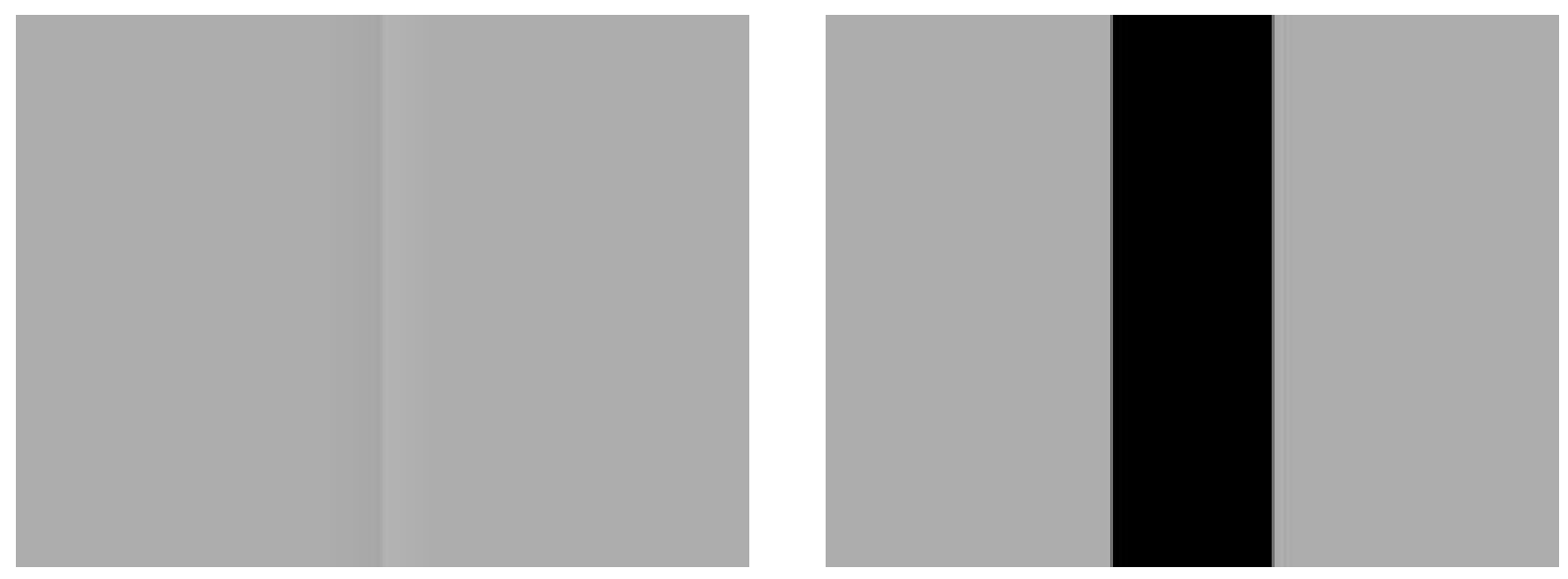

Figure 2: Craik-O'Brien-Cornsweet illusion. The left part seems darker than the right part (left figure), but when the transient region is masked out this illusion disappears (right figure). This example questions the position that the bright sun illusion arises (exclusively) at a semantic level due to our predisposition to judge sunlight as brightest. But what could possibly be Nature's "purpose" to trigger such illusions a priori?

multiple of this, $S(x) \sigma(x)$, for each $x$, is referred to as a section of the fibred space. We introduce a connection via a covariant derivative operator $\mathscr{D}$ —as a genuine extension of the covariant derivative used in the foregoingwhich we subject to the usual requirement posed by the product rule:

$$
\mathscr{D}(S(x) \sigma(x))=\mathscr{D} S(x) \sigma(x)+S(x) \mathscr{D} \sigma(x) .
$$

We then identify $\mathscr{D} S(x)=\nabla S(x)$, the usual gradient of the scalar function $S$ evaluated at $x$. The unit $\sigma(x)$ is treated as a local basis vector for the one-dimensional fibre at $x$, and so the quantity $\mathscr{D} \sigma(x)$ must itself be a section, i.e. a local multiple of the basis vector $\sigma(x)$. In this way we obtain a local gauge field $A$ (a covector field) with amplitude $A(x)$ at $x$ :

$$
\mathscr{D} \sigma(x)=A(x) \sigma(x) .
$$

All in all, the components of the covariant derivative of the signal relative to a local basis can be written as

$$
\mathscr{D}_{\mu}(S(x) \sigma(x)) \mathbf{e}^{\mu}(x)=D_{\mu}^{A} S(x) \sigma(x) \mathbf{e}^{\mu}(x),
$$

with $^{2}$

$$
D_{\mu}^{A} S(x)=\left(\partial_{\mu}+A_{\mu}(x)\right) S(x) .
$$

Effectively the whole procedure thus boils down to what is known as the "principle of minimal substitution" in physics, whereby one replaces ordinary differentiation of the signal function (i.e. the coefficient of the local basis section $\sigma(x)$ ), by covariant differentiation ${ }^{3}: \partial_{\mu} S(x) \rightarrow\left(\partial_{\mu}+A_{\mu}(x)\right) S(x)$.

It remains an outstanding problem how the visual system gauges itself after exposure to a given signal. In any case, prolonged exposure to a stationary scene $(S(x)>0$ independent of time) and deprived from the possibility to make saccadic eye movements "nullifies" one's percept completely, which can be understood as an adaptation mechanism in which the input stimulus has somehow evolved towards a "covariantly constant" steady state, i.e. $D_{\mu}^{A} S(x)=0$, inducing a gauge field

$$
A_{\mu}(x)=-\partial_{\mu} \ln S(x) .
$$

Intuitively it makes sense from an economic and ecological point of view to consider a stationary background stimulus as "void".

Our paradigmatic equation, Eq. (13), can now be modified so as to account for a background gauge field through minimal substitution of the Laplace-Beltrami operator:

$$
\partial_{t} S_{t}-\Delta_{g}^{A} S_{t}=0
$$

\footnotetext{
${ }^{2}$ Additional $\Gamma$-corrections will be needed for non-scalar signals, recall Section 2.1, e.g. $D_{\mu}^{A, \Gamma} v^{v}(x)=\left(\delta_{\rho}^{v}\left(\partial_{\mu}+A_{\mu}(x)\right)+\Gamma_{\rho \mu}^{v}(x)\right) v^{\rho}(x)$.

${ }^{3}$ In physics, however, one typically considers Hermitean operators $i \partial_{\mu}$, respectively $i \partial_{\mu}+A_{\mu}$.
} 
with self-dual gauge adapted Laplace-Beltrami operator ${ }^{4}$

$$
\Delta_{g}^{A}=g^{\mu v}\left(D_{\mu}-A_{\mu}\right)\left(D_{v}+A_{v}\right)=D_{A}^{\mu} D_{\mu}^{A} .
$$

In this case ordinary partial derivatives have been replaced by ones that are covariant in both domain as well as codomain: $\partial_{\mu} \rightarrow D_{\mu} \rightarrow D_{\mu}^{A}, \partial^{\mu} \rightarrow D^{\mu} \rightarrow D_{A}^{\mu}$, with $D_{A}^{\mu}=g^{\mu \nu} D_{v}^{-A}$. The reason for this definition is that it extends the property of the null-gauged Laplace-Beltrami operator as the functional derivative

$$
\frac{1}{2} \frac{\delta}{\delta u} \int g^{\mu v}(\xi) D_{\mu} u(\xi) D_{v} u(\xi) D \xi=-\Delta_{g} u
$$

to the case involving a non-trivial gauge field $A_{\mu}$ :

$$
\frac{1}{2} \frac{\delta}{\delta u} \int g^{\mu v}(\xi) D_{\mu}^{A} u(\xi) D_{v}^{A} u(\xi) D \xi=-\Delta_{g}^{A} u .
$$

Combined with reparametrization of the codomain we find the generalization of Eq. (16):

$$
\partial_{t} S_{t}-\Delta_{g}^{A} S_{t}-\mu\left\|\nabla^{A} S_{t}\right\|_{g}^{2}=0,
$$

with $\left\|\nabla^{A} u\right\|_{g}^{2}=g^{\mu v} D_{\mu}^{A} u D_{v}^{A} u=D_{-A}^{\mu} u D_{\mu}^{A} u$. This is our main result. The conjecture is that it underlies many instances of multi-resolution systems in physics, biology, and engineering sciences.

\subsection{Examples}

\subsubsection{Human Vision}

In the following example we take $m=n=2$. (There will consequently be no need to distinguish between Greek and Latin spatial indices, since embedded surface and embedding space coincide.)

Elsewhere it has been argued that the retino-cortical pathway of the human visual system can be understood from a differential geometric point of view in terms of a certain conformal metric transform that reflects the foveal properties of the human eye $[9,10]$. The proposed metric takes the following form in polar coordinates ${ }^{5}$, $\left(\xi^{1}, \xi^{2}\right)=(r, \theta)$ :

$$
g_{\mu v}=\left(\frac{r_{0}}{r}\right)^{2} \eta_{\mu v}
$$

in which $r_{0}$ is a biological size parameter characteristic of the central fovea, and $\eta_{\mu \nu}$ the Euclidean metric,

$$
\eta_{\mu v}=\left(\begin{array}{cc}
1 & 0 \\
0 & r^{2}
\end{array}\right)
$$

This conformal metric reflects the fact that, to reasonable approximation, the human visual system has a foveal bias (objects near the foveal point are magnified inversely proportional to eccentricity), but is otherwise isotropic (angular relations are not affected). The corresponding Laplace-Beltrami operator $\Delta_{g}$ turns out to be proportional to the Euclidean Laplacian $\Delta_{\eta}$, which should be regarded as a peculiarity of a conformal metric in $n=2$ dimensions (it no longer holds when $n>2$ ):

$$
\Delta_{g}=\left(\frac{r}{r_{0}}\right)^{2} \Delta_{\eta} .
$$

Consequently the generating equation, Eq. (19) based on metric Eq. (20) and with $\gamma=\operatorname{id}$ and $A_{\mu}=0$, is given by

$$
\partial_{t} S_{t}-\left(\frac{r}{r_{0}}\right)^{2} \Delta_{\eta} S_{t}=0 .
$$

\footnotetext{
${ }^{4}$ This definition differs from the one proposed by Georgiev, which fails to be self-dual [11].

${ }^{5}$ At this level of rigor we ignore the singularity at the origin, but cf. [10].
} 


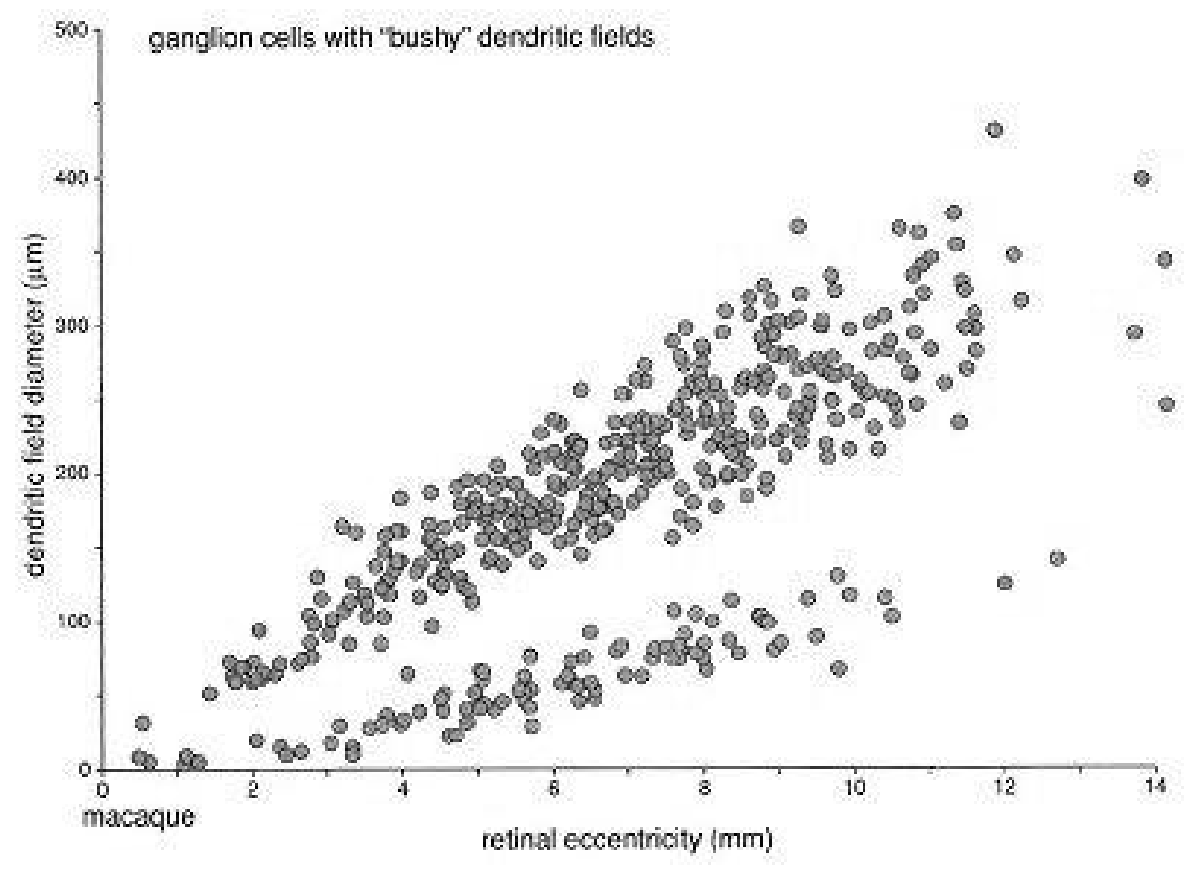

Figure 3: Dendritic field diameter-a direct measure for receptive field size-versus retinal eccentricity in macaque retina. After Watanabe and Rodieck [25, 30].

A natural scale reparametrization presents itself, viz.

$$
\left(\frac{r}{r_{0}}\right)^{2} t=s,
$$

after which the reparametrized system appears homogeneous ${ }^{6}$. Recall that $t$ and $s$ are quadratic scales, thus one expects biological receptive field sizes to scale linearly with eccentricity $r / r_{0}$. (Resolution limitations are not taken into account here.) There exists ample evidence in support of this, cf. Fig. 3. The structure of the human retina and its retinotopic mapping onto the striate cortex (also known as cortical area V1) reflects the above-sketched geometric structure remarkably well, and so does ample psychophysical evidence [3, 25].

Let us now briefly return to the parametrization issue, recall Section 2.3. The Weber-Fechner law is a wellknown psychophysical law that applies to various perceptual modalities. It states that the intensity of the percept is a logarithmic function of the intensity of the physical stimulus. Here we consider the relation between apparent brightness $U_{t}$ and retinal irradiation $S_{t}$. The Weber-Fechner law is a direct consequence of Eq. (16) if we take $\mu$ to be a positive constant. Indeed, in this case one readily finds $\gamma\left(U_{t}\right)$ to be an exponential function (defined up to a pair of integration constants). If we impose the boundary conditions $\gamma(-\infty)=0$ and $\gamma(\infty)=\infty$ (so that $S_{t}$ is positive definite), and set $\gamma(0)=\gamma_{0}$ for some constant $\gamma_{0}>0$, we obtain

$$
U_{t}=\frac{1}{\mu} \ln \frac{S_{t}}{\gamma_{0}} .
$$

A least noticeable perceptual difference $d U_{t}$ then corresponds to a logarithmic increment $\mu^{-1} d S_{t} / S_{t}$ of photon flux. One could interpret $\mu^{-1}$ as a psychophysical unit of dimension for the quantity $U_{t}$. The constant $\gamma_{0}$ may be adaptive to ambient light conditions.

The Weber-Fechner law holds only within an interval of physical photon fluxes of a few orders of magnitude. In general one will need to account for threshold and saturation phenomena. A way to achieve this is to replace

\footnotetext{
${ }^{6}$ Homegeneity in fact holds almost everywhere in the sense that space remains flat except at the foveal centre, at which the Ricci curvature tensor degenerates. This singularity can be removed at the expense of introducing global curvature with appreciable magnitude in the fovea centralis [10].
} 
the unbounded mapping $\gamma$ above by $\gamma_{\chi}=\gamma \circ \chi$ for some suitably chosen psychophysical function $\chi$, such that $\chi^{-1}$ is bounded and monotonic, say $\chi^{-1}: \mathbb{R} \rightarrow(0,1)$. This amounts to a replacement of the nonlinearity coefficient $\mu \rightarrow \mu_{\chi}=\mu \chi^{\prime}+\chi^{\prime \prime} / \chi^{\prime}$.

Finally, it may be worth exploring the feasibility of the general equation, Eq. (19), as a generic model for "early vision", accounting for retino-cortical mapping (via the stipulated conformal metric transform), nonlinear signal transduction (via suitable codomain reparametrization), and illusionary "lightness", or perceived brightness, in relation to the actual physical stimulus (via adaptation mediated by some gauge field). As far as the latter aspect is concerned we find ourselves still largely in the dark. Most likely the gauge is fixed by some dynamic, global adaptation mechanism (point entities such as they occur in Eq. (17) are physically void). This supports the intriguing conjecture, raised by Koenderink [17], that the brain can be understood as a "geometry engine".

\subsubsection{High Angular Resolution Diffusion Imaging}

In the following example we take $m=2, n=3$, and identify $\Omega^{2}$ with the unit sphere embedded in Euclidean 3 -space, $\mathbb{R}^{3}$. This case is of considerable interest in high angular resolution diffusion imaging (HARDI), a noninvasive magnetic resonance imaging technique for mapping local water diffusivity profiles in vivo [1, 2, 20]. It is conjectured that such profiles convey important information on the architecture of fibrous tissues, such as brain white matter (axons) and muscles, since water diffusion is facilitated in the direction of the underlying fibres.

A typical HARDI acquisition entails a large number of signal attenuation measurements in different directions. Various representations have been proposed in the literature to handle such multi-directional data. Typically these take the form of (square-integrable) scalar functions on the unit sphere.

Let therefore $S: \Omega^{2} \rightarrow \mathbb{R}^{3}$ denote a given raw HARDI signal confined to the unit sphere $\Omega^{2}:\|x\|=1, x \in \mathbb{R}^{3}$. $\Omega$ may be parametrized using two coordinates, $\xi^{\mu}, \mu=1,2$, say. An obvious choice would be the polar angles $\xi=\left(\xi^{1}, \xi^{2}\right)=(\theta, \phi) \in[0, \pi] \times[0,2 \pi)$, given in terms of 3D Cartesian coordinates $x^{i}, i=1,2,3$, by

$$
\left\{\begin{array}{l}
x^{1}=\sin \theta \cos \phi \\
x^{2}=\sin \theta \sin \phi \\
x^{3}=\cos \theta
\end{array}\right.
$$

The components of the Riemannian metric for the unit sphere embedded in Euclidean 3-space $\mathbb{R}^{3}$ are given by Eq. (1). Using polar and Cartesian coordinates for $\Omega^{2}$ and $\mathbb{R}^{3}$, respectively, we obtain the familiar matrix representations

$$
g_{\mu \nu}=\left(\begin{array}{cc}
1 & 0 \\
0 & \sin ^{2} \theta
\end{array}\right) \quad \text { resp. } \quad \eta_{i j}=\left(\begin{array}{lll}
1 & 0 & 0 \\
0 & 1 & 0 \\
0 & 0 & 1
\end{array}\right) .
$$

In particular, $D \xi=\sqrt{g} d \xi^{1} d \xi^{2}=\sin \theta d \theta d \phi$ is the invariant volume element on $\Omega^{2}$.

We may think of $\Omega^{2}$ as a "fibre" over the Euclidean space $\mathbb{R}^{3}$ at some implicit base point $x \in \mathbb{R}^{3}$. More specifically, in the context of HARDI, $\Omega$ is the domain of definition of the diffusive attenuation signal $S$ or diffusion coefficient $D$ related via the Stejskal-Tanner formula [22, 28]:

$$
S(\theta, \phi)=S_{0} \exp (-b D(\theta, \phi))
$$

In what follows we shall consider the scale space extension of $S$, but this may be equally well replaced by any other $L^{2}\left(\Omega^{2}\right)$-function, notably $D$, or by derived probabilistic quantities such Tuch's orientation distribution function (ODF) [29], the higher order diffusion tensor model and the diffusion orientation transform (DOT) by Özarslan et al. [22, 23], and the diffusion tensor distribution model by Jian et al. [15]. See also Descoteaux et al. $[5,6]$ and Hess et al. [14].

Consider the scale space representation $S_{t}$ of $S$ derived in Section 2.2. The (dimensionless) parameter $t \in \mathbb{R}^{+}$ now controls (inverse) angular resolution. Expressed in terms of spherical coordinates it is natural to decompose the functions $S_{t}$ and $S$ relative to the orthonormal basis of spherical harmonics, since this diagonalizes the LaplaceBeltrami operator:

$$
\Delta_{g} Y_{\ell}^{m}=-\ell(\ell+1) Y_{\ell}^{m}
$$


for any $\ell \in \mathbb{Z}_{0}^{+}$and $m \in\{-\ell,-\ell+1, \ldots, \ell-1, \ell\}$. We employ the following convention for spherical harmonics, in which $P_{\ell}^{m}$ are the associated Legendre polynomials ${ }^{7}$ :

$$
\begin{aligned}
Y_{\ell}^{m}(\theta, \phi) & =\sqrt{\frac{(2 \ell+1)(\ell-m) !}{4 \pi(\ell+m) !}} e^{i m \phi} P_{\ell}^{m}(\cos \theta) . \\
P_{\ell}^{m}(z) & =\frac{(-1)^{m}}{2^{\ell} \ell !}\left(1-z^{2}\right)^{\frac{m}{2}} \frac{d^{\ell+m}}{d z^{\ell+m}}\left(z^{2}-1\right)^{\ell}
\end{aligned}
$$

with $-1 \leq z \leq 1$. Setting

$$
S(\theta, \phi)=\sum_{\ell, m}^{*} c_{\ell m}(0) Y_{\ell}^{m}(\theta, \phi),
$$

in which the asterisk indicates summation over the effective indices $\ell \in \mathbb{Z}_{0}^{+}$and $m \in\{-\ell,-\ell+1, \ldots, \ell-1, \ell\}$, yields

$$
S_{t}(\theta, \phi)=\sum_{\ell, m}^{*} c_{\ell m}(t) Y_{\ell}^{m}(\theta, \phi)
$$

with

$$
c_{\ell m}(t)=e^{-t \ell(\ell+1)} c_{\ell m}(0) .
$$

Notice the analogy with the $e^{-t\|\omega\|^{2}}$-attenuation of high frequency components of scalar images in the Euclidean plane under Gaussian blurring.

The coefficients in Eq. (31) are determined as follows:

$$
c_{\ell m}(0)=\int_{0}^{2 \pi} \int_{0}^{\pi} S(\theta, \phi) Y_{\ell}^{-m}(\theta, \phi) \sin \theta d \theta d \phi,
$$

by virtue of $Y_{\ell}^{-m}=\left(Y_{\ell}^{m}\right)^{*}$ and orthonormality:

$$
\int_{0}^{2 \pi} \int_{0}^{\pi} Y_{\ell}^{m}(\theta, \phi)^{*} Y_{\ell^{\prime}}^{m^{\prime}}(\theta, \phi) \sin \theta d \theta d \phi=\delta_{m m^{\prime}} \delta_{\ell \ell^{\prime}} .
$$

Eqs. (32-34) summarize the operational scheme for extending a raw HARDI signal into a continuous scale space family defined implicitly via minimization of Eq. (8). The required integrals over the sphere can be numerically approximated in the standard way, e.g. by employing a regular sampling of directions on vertices of a tessellated icosahedron with a corresponding numerical integration measure induced by the areas of the polygons defined by the dual tessellation.

The asymptotic cases are as expected:

$$
\begin{aligned}
\lim _{t \rightarrow 0^{+}} S_{t}(\theta, \phi) & =S(\theta, \phi), \\
\lim _{t \rightarrow \infty} S_{t}(\theta, \phi) & =\frac{1}{4 \pi} \int_{0}^{2 \pi} \int_{0}^{\pi} S(\theta, \phi) \sin \theta d \theta d \phi,
\end{aligned}
$$

i.e., in the hypothetical limit of infinite angular resolution Eqs. (32-34) reproduce the raw signal, whereas the limit of vanishing angular resolution corresponds to a complete averaging of this raw signal over the sphere. Similar Tikhonov regularization procedures of unit sphere HARDI functions have been proposed in the literature by Descoteaux et al. [6] and by Hess et al. [14]. Fig. 4 illustrates Eq. (32) on a test image.

\section{Summary}

We have adapted the linear scale space paradigm to the local geometry of the image domain (base manifold) as well as to the potentially local nature of codomain gauge (fibre bundle over the base manifold). The theory has been illustrated with two examples, viz. regularization of high angular resolution diffusion imaging (spherical base manifold geometry), and modeling of the retino-cortical pathway in human vision (conformal metric transform). In the latter case we have pointed at the potential relevance of a fibre bundle construct, but detailed mechanisms are hitherto unknown. Much research will be needed to understand the role local of adaptation mechanisms in the psychophysics of human perception.

\footnotetext{
${ }^{7} \mathrm{Cf}$. functions.wolfram.com for further properties of $Y_{\ell}^{m}$ and $P_{\ell}^{m}$.
} 


\section{acknowledgement}

The Netherlands Organisation for Scientific Research (NWO) is gratefully acknowledged for financial support.

\section{References}

[1] P. J. Basser, J. Mattiello, and D. Le Bihan. Estimation of the effective self-diffusion tensor from the NMR spin echo. Journal of Magnetic Resonance, 103:247-254, 1994.

[2] P. J. Basser, J. Mattiello, and D. Le Bihan. MR diffusion tensor spectroscopy and imaging. Biophysics Journal, 66(1):259-267, 1994.

[3] P. Bijl. Aspects of Visual Contrast Detection. PhD thesis, Utrecht University, Department of Physics, Utrecht, The Netherlands, May 81991.

[4] B. Burgeth, S. Didas, and J. Weickert. Relativistic scale-spaces. In R. Kimmel, N. Sochen, and J. Weickert, editors, Scale Space and PDE Methods in Computer Vision: Proceedings of the Fifth International Conference, Scale-Space 2005, Hofgeismar, Germany, volume 3459 of Lecture Notes in Computer Science, pages $1-12$, Berlin, April 2005. Springer-Verlag.

[5] M. Descoteaux, E. Angelino, S. Fitzgibbons, and R. Deriche. Apparent diffusion coefficients from high angular resolution diffusion imaging: Estimation and applications. Magnetic Resonance in Medicine, 56(2):395-410, 2006.

[6] M. Descoteaux, E. Angelino, S. Fitzgibbons, and R. Deriche. Regularized, fast, and robust analytical Q-ball imaging. Magnetic Resonance in Medicine, 58(3):497-510, 2007.

[7] R. Duits, L. Florack, J. de Graaf, and B. ter Haar Romeny. On the axioms of scale space theory. Journal of Mathematical Imaging and Vision, 20(3):267-298, 2004.

[8] L. M. J. Florack. Image Structure, volume 10 of Computational Imaging and Vision Series. Kluwer Academic Publishers, Dordrecht, The Netherlands, 1997.

[9] L. M. J. Florack. A geometric model for cortical magnification. In S.-W. Lee, H. H. Bülthoff, and T. Poggio, editors, Biologically Motivated Computer Vision: Proceedings of the First IEEE International Workshop, BMCV 2000 (Seoul, Korea, May 2000), volume 1811 of Lecture Notes in Computer Science, pages 574-583, Berlin, May 2000. Springer-Verlag.

[10] L. M. J. Florack. Modeling foveal vision. In F. Sgallari, A. Murli, and N. Paragios, editors, Scale Space and Variational Methods in Computer Vision: Proceedings of the First International Conference, SSVM 2007, Ischia, Italy, volume 4485 of Lecture Notes in Computer Science, pages 919-928, Berlin, May-June 2007. Springer-Verlag.

[11] T. Georgiev. Covariant derivatives and vision. In A. Leonardis, H. Bischof, and A. Prinz, editors, Proceedings of the Ninth European Conference on Computer Vision (Graz, Austria, May 2006), volume 3951-3954 of Lecture Notes in Computer Science, pages 56-69, Berlin, Heidelberg, 2006. Springer-Verlag.

[12] B. M. ter Haar Romeny, editor. Geometry-Driven Diffusion in Computer Vision, volume 1 of Computational Imaging and Vision Series. Kluwer Academic Publishers, Dordrecht, 1994.

[13] B. M. ter Haar Romeny. Front-End Vision and Multi-Scale Image Analysis: Multi-Scale Computer Vision Theory and Applications, written in Mathematica, volume 27 of Computational Imaging and Vision Series. Kluwer Academic Publishers, Dordrecht, The Netherlands, 2003.

[14] C. P. Hess, P. Mukherjee, E. T. Tan, D. Xu, and D. B. Vigneron. Q-ball reconstruction of multimodal fiber orientations using the spherical harmonic basis. Magnetic Resonance in Medicine, 56:104-117, 2006.

[15] B. Jian, B. C. Vemuri, E. Özarslan, P. R. Carney, and T. H. Mareci. A novel tensor distribution model for the diffusion-weighted MR signal. NeuroImage, 37:164-176, 2007. 
[16] J. J. Koenderink. The structure of images. Biological Cybernetics, 50:363-370, 1984.

[17] J. J. Koenderink. The brain a geometry engine. Psychological Research, 52:122-127, 1990.

[18] J. J. Koenderink. Image space. In R. Ablamwicz, editor, Clifford Algebras; Applications to Mathematics, Physics, and Engineering, pages 577-596. Birkhaüser, Boston, 2004.

[19] J. J. Koenderink and A. D. van Doorn. Image processing done right. In A. Heyden, G. Sparr, M. Nielsen, and P. Johansen, editors, Proceedings of the Seventh European Conference on Computer Vision (Copenhagen, Denmark, May-June 2002), volume 2350-2353 of Lecture Notes in Computer Science, pages 158-172, Berlin, 2002. Springer-Verlag.

[20] D. Le Bihan, J.-F. Mangin, C. Poupon, C. A. Clark, S. Pappata, N. Molko, and H. Chabriat. Diffusion tensor imaging: Concepts and applications. Journal of Magnetic Resonance Imaging, 13:534-546, 2001.

[21] T. Lindeberg. Scale-Space Theory in Computer Vision. The Kluwer International Series in Engineering and Computer Science. Kluwer Academic Publishers, Dordrecht, The Netherlands, 1994.

[22] E. Özarslan and T. H. Mareci. Generalized diffusion tensor imaging and analytical relationships between diffusion tensor imaging and high angular resolution imaging. Magnetic Resonance in Medicine, 50(5):955$965,2003$.

[23] E. Özarslan, T. M. Shepherd, B. C. Vemuri, S. J. Blackband, and T. H. Mareci. Resolution of complex tissue microarchitecture using the diffusion orientation transform (DOT). NeuroImage, 31:1086-1103, 2006.

[24] P. Perona and J. Malik. Scale-space and edge detection using anisotropic diffusion. IEEE Transactions on Pattern Analysis and Machine Intelligence, 12(7):629-639, July 1990.

[25] R. W. Rodieck. The First Steps in Seeing. Sinauer Associates, Inc., Sunderland, Massachusetts, 1998.

[26] M. Spivak. Differential Geometry, volume 1-5. Publish or Perish, Berkeley, 1975.

[27] J. Sporring, M. Nielsen, L. M. J. Florack, and P. Johansen, editors. Gaussian Scale-Space Theory, volume 8 of Computational Imaging and Vision Series. Kluwer Academic Publishers, Dordrecht, The Netherlands, 1997.

[28] E. O. Stejskal and J. E. Tanner. Spin diffusion measurements: Spin echoes in the presence of a timedependent field gradient. Journal of Computational Physics, 42:288-292, 1965.

[29] D. S. Tuch. Q-ball imaging. Magnetic Resonance in Medicine, 52:1358-1372, 2004.

[30] M. Watanabe and R. W. Rodieck. Parasol and midget ganglion cells of the primate retina. Journal of Comparative Neurology, 289:434-454, 1989.

[31] J. A. Weickert. Anisotropic Diffusion in Image Processing. ECMI Series. Teubner, Stuttgart, January 1998. 


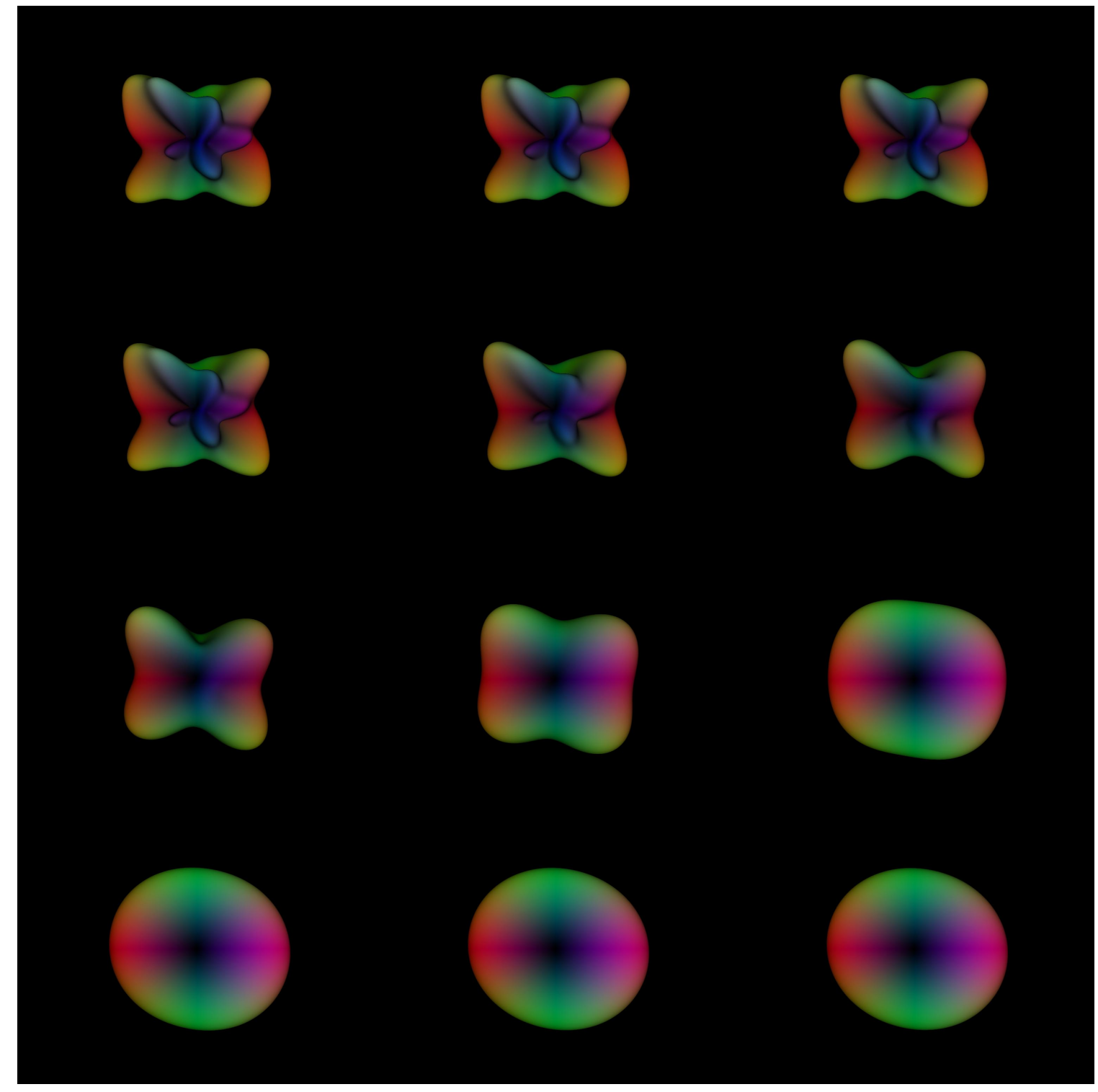

Figure 4: A scalar function on the unit sphere, with intensity displayed as radial distince from the origin, viewed at (exponentially) increasing values of the inverse angular resolution parameter $t$. 
PREVIOUS PUBLICATIONS IN THIS SERIES:

\begin{tabular}{|c|c|c|c|}
\hline Number & Author(s) & Title & Month \\
\hline $10-59$ & $\begin{array}{l}\text { A. Thornton } \\
\text { T. Weinhart } \\
\text { O. Bokhove } \\
\text { B. Zhang } \\
\text { D.M. van der Sar } \\
\text { K. Kumar } \\
\text { M. Pisarenco } \\
\text { M. Rudnaya } \\
\text { V. Savcenco } \\
\text { J. Rademacher } \\
\text { J. Zijlstra } \\
\text { A. Szabelska } \\
\text { J. Zyprych } \\
\text { M. van der Schans } \\
\text { V. Timperio } \\
\text { F. Veerman }\end{array}$ & $\begin{array}{l}\text { Modeling and optimization } \\
\text { of algae growth }\end{array}$ & Oct. 'Io \\
\hline $10-60$ & $\begin{array}{l}\text { V. Savcenco } \\
\text { B. Haut }\end{array}$ & $\begin{array}{l}\text { Multirate integration of a } \\
\text { European power system } \\
\text { network model }\end{array}$ & Oct. 'Iо \\
\hline IO-6I & $\begin{array}{l}\text { V. Savcenco } \\
\text { B. Haut } \\
\text { E.J.W. ter Maten } \\
\text { R.M.M. Mattheij }\end{array}$ & $\begin{array}{l}\text { Time domain simulation } \\
\text { of power systems with } \\
\text { different time scales }\end{array}$ & Oct. 'Iо \\
\hline $10-62$ & $\begin{array}{l}\text { D.J. Bekers } \\
\text { S.J.L. van Eijndhoven }\end{array}$ & $\begin{array}{l}\text { Spectral analysis of } \\
\text { integral-differential } \\
\text { operators applied in linear } \\
\text { antenna modeling }\end{array}$ & Oct. 'IO \\
\hline $10-63$ & L.M.J. Florack & $\begin{array}{l}\text { Scale space } \\
\text { representations locally } \\
\text { adapted to the geometry of } \\
\text { base and target manifold }\end{array}$ & Oct. 'IO \\
\hline
\end{tabular}

